\title{
Cavity problems in discontinuous media
}

\author{
by \\ Disson dos Prazeres and Eduardo V. Teixeira
}

\begin{abstract}
We study cavitation type equations, $\operatorname{div}\left(a_{i j}(X) \nabla u\right) \sim \delta_{0}(u)$, for bounded, measurable elliptic media $a_{i j}(X)$. De Giorgi-Nash-Moser theory assures that solutions are $\alpha$-Hölder continuous within its set of positivity, $\{u>0\}$, for some exponent $\alpha$ strictly less than one. Notwithstanding, the key, main result proven in this paper provides a sharp Lipschitz regularity estimate for such solutions along their free boundaries, $\partial\{u>0\}$. Such a sharp estimate implies geometric-measure constrains for the free boundary. In particular, we show that the non-coincidence $\{u>0\}$ set has uniform positive density and that the free boundary has finite $(n-\varsigma)$-Hausdorff measure, for a universal number $0<\varsigma \leq 1$.
\end{abstract}

AMS Subject Classifications: 35B65, 35R35.

\section{Introduction}

Given a Lipschitz bounded domain $\Omega \subset \mathbb{R}^{n}$, a bounded measurable elliptic matrix $a_{i j}(X)$, i.e. a symmetric matrix with varying coefficients satisfying the $(\lambda, \Lambda)$-ellipticity condition

$$
\lambda \mathrm{Id} \leq a_{i j}(X) \leq \Lambda \mathrm{Id},
$$

and a nonnegative boundary data $\varphi \in L^{2}(\partial \Omega)$, we are interested in studying local minimizers $u$ of the discontinuous functional

$$
\mathcal{F}(u)=\int_{\Omega}\left\{\frac{1}{2}\left\langle a_{i j}(X) \nabla u, \nabla u\right\rangle+\chi_{\{u>0\}}\right\} d X \rightarrow \min ,
$$

among all competing functions $u \in H_{\varphi}^{1}(\Omega):=\left\{u \in H^{1}(\Omega) \mid \operatorname{Trace}(u)=\varphi\right\}$.

The variational problem set in (1.2) appears in the mathematical formulation of a great variety of models: jet flows, cavity problems, Bernoulli problems, free transmission problems, optimal designs, just to cite few. Its mathematical treatment has been extensively developed since the epic marking work of Alt and Caffarelli [1]. The program for studying minimization problems for discontinuous functionals of the form (1.2) is nowadays well established in the literature. Existence of minimizer follows by classical considerations. Any minimum is nonnegative provided the boundary data is 
nonnegative. Minimizers satisfy, in the distributional sense, the Euler-Lagrange equation

$$
\operatorname{div}\left(a_{i j}(X) \nabla u\right)=\mu,
$$

where $\mu$ is a measure supported along the free boundary. In particular a minimum of the functional $\mathcal{F}$ is $a$-harmonic within its positive set, i.e.,

$$
\operatorname{div}\left(a_{i j}(X) \nabla u\right)=0, \quad \text { in }\{u>0\} \cap \Omega .
$$

By pure energy considerations, one proves that minimizers grow linearly alway from their free boundaries. Finally, if $a_{i j}$ are, say, Hölder continuous, then the free boundary $\partial\{u>0\}$ is of class $C^{1, \alpha}$ up to a possible negligible singular set. In such a scenario, the free boundary condition

$$
\left\langle a_{i j}(\xi) \nabla u(\xi), \nabla u(\xi)\right\rangle=\text { Const. }
$$

then holds in the classical sense along the regular part of the free boundary, in particular for all $\xi \in \partial_{\text {red }}\{u>0\} \cap \Omega$.

A decisive, key step, though, required in the program for studying variational problems of the form [1.2), concerns Lipschitz estimates of minimizers. However, if no further regularity assumptions upon the coefficients $a_{i j}(X)$ is imposed, even $a$-harmonic functions, $\operatorname{div}\left(a_{i j}(X) \nabla h\right)=0$, may fail to be Lipschitz continuous. That is, the universal Hölder continuity exponent granted by De Giorgi-Nash-Moser regularity theory may be strictly less than 1, even for two-dimensional problems. Such a technical constrain makes the study of local minima to (1.2) in discontinuous media rather difficult from a rigorous mathematical viewpoint.

The above discussion brings us to the main goal of this present work. Even though it is hopeless to obtain gradient bounds for minimizers of functional $(1.2)$ in $\Omega$, we shall prove that, any minimum is universally Lipschitz continuous along its free boundary, $\partial\{u>0\} \cap \Omega$, see [25, 26] for improved estimates that hold only along (nonphysical) free boundaries, see also [20]. Such an estimate is strong enough to carry on a geometric-measure analysis near the free boundary, which in particular implies that the non-coincidence set has uniform positive density and that the free boundary has finite $(n-\varsigma)$-Hausdorff measure, for a universal number $0<\varsigma \leq 1$. We shall establish the following result:

Theorem 1.1. Let $u$ be a nonnegative local minimum of the functional 1.2 and $Z_{0} \in$ $\partial\{u>0\} \cap \Omega$ be a generic interior free boundary point. Then

$$
C^{-1} r \leq \sup _{B_{r}\left(Z_{0}\right)} u \leq C r,
$$

for a constant $C>0$ depending only on dimension, ellipticity constants and $\|u\|_{L^{2}(\Omega)}$. In particular, for another universal constant $\theta>0$,

$$
\mathscr{L}^{n}\left(\{u>0\} \cap B_{r}\left(Z_{0}\right)\right) \geq \theta r^{n},
$$


for all $0<r \ll 1$. Furthermore there is a universal constant $0<\varsigma \leq 1$ such that

$$
\operatorname{dim}_{\mathscr{H}}(\partial\{u>0\}) \leq n-\varsigma,
$$

where $\operatorname{dim}_{\mathscr{H}}(E)$ means the Hausdorff dimension to the set $E$.

In this paper we shall develop a more general analysis as to contemplate singular approximations of the minimization problem (1.2). Let $\beta \in L^{\infty}(\mathbb{R})$ be a bounded function supported in the unit interval $[0,1]$. For each $\varepsilon>0$, we define the integral preserving, $\varepsilon$-perturbed potential:

$$
\beta_{\varepsilon}(t):=\frac{1}{\varepsilon} \beta\left(\frac{t}{\varepsilon}\right),
$$

which is now supported in $[0, \varepsilon]$. Such a sequence of potentials converges in the distributional sense to $\int \beta$ times the Dirac measure $\delta_{0}$. Consider further

$$
B_{\varepsilon}(\xi)=\int_{0}^{\xi} \beta_{\varepsilon}(t) d t \rightarrow\left(\int \beta(s) d s\right) \cdot \chi_{\{\xi>0\}},
$$

in the distributional sense. We now look at local minimizers $u_{\varepsilon}$ to the variational problem

$$
\mathcal{F}_{\mathcal{\varepsilon}}(u)=\int_{B_{1}}\left\{\frac{1}{2}\left\langle a_{i j}(X) \nabla u, \nabla u\right\rangle+B_{\mathcal{\varepsilon}}(u)\right\} d X \rightarrow \min ,
$$

among all competing functions $u \in H_{\varphi}^{1}(\Omega):=\left\{u \in H^{1}(\Omega) \mid \operatorname{Trace}(u)=\varphi\right\}$. There is a large literature on such a class of singularly perturbed equations, see for instance [3, 8, 9, 11, 18, 19, 22]. It is well established that the functional $\mathcal{F}$ defined in (1.2) can be recovered by letting $\varepsilon$ go to zero in (1.6). For each $\varepsilon$ fixed though, minimizers of the functional $\mathcal{F}_{\mathcal{E}}$ is related to a number of other physical problems, such as high energy activations and the theory of flame propagation. Hence, from the applied point of view, it is more appealing to indeed study the whole family of functionals $\left(\mathcal{F}_{\varepsilon}\right)_{0<\varepsilon<1}$. We also mention that the study of minimization problem (1.6) with no continuity assumption on the coefficients is also motivated by several branch of applications, for instance in homogenization theory, composite materials, etc.

We should also mention the connections this present work has with the theory of free phase transmission problems. This class of problems appears, for instance, in the system of equations modeling an ice that melts submerged in a heated inhomogeneous medium. For problems modeled within an organized medium (say Hölder continuous coefficients), monotonicity formula [5] yields Lipschitz estimates for solutions. However, by physical interpretations of the model, it is natural to consider the problem within discontinuous media. Under such an adversity (monotonicity formula is no longer available), Lipschitz estimate along the free boundary has been an important open problem within that theory, see [2] for discussion. However, if we further assume in the model that the temperature of the ice remains constant, which is reasonable in very low temperatures, then free phase transmission problems fit into the mathematical 
formulation of this present article; and a Lipschitz estimate becomes available by our main result.

We conclude this Introduction by mentioning that the improved, sharp regularity estimate we establish in this work holds true in much more generality. Our approach to obtain Lipschitz estimate along the free boundary extends directly to degenerate discontinuous functionals of the form

$$
\int F(X, u, \nabla u) d X \rightarrow \min .
$$

where

$$
F(X, u, \xi) \sim|\xi|^{p-2} A(X) \xi \cdot \xi+f(X)\left(u^{+}\right)^{m}+Q(X) \cdot \chi_{\{u>0\}},
$$

with $A(X)$ bounded, measurable elliptic matrix, $f \in L^{q}(\Omega), q>n, 1 \leq m<p$ and $Q$ is bounded away from zero and infinity, see [12, 16]. Indeed, the proof designed herein is purely nonlinear and uses solely the Euler-Lagrange equation associated to the minimization problem (1.6). Hence, nonvariational cavitation problems, as well as parabolic versions of such models can also be tackled by our methods.

\section{Preliminaries}

In this Section we gather some results and tools available for the analysis of minimizers to the functional (1.6) (and also to the functional (1.2)). The results stated herein follow by methods and approaches available in the literature. We shall briefly comment on the proofs, for the readers' convenience.

Theorem 2.1 (Existence of minimizers). For each $\varepsilon>0$ fixed, there exists at least one minimizer $u_{\varepsilon} \in H_{\varphi}^{1}(\Omega)$ to the function (1.6). Furthermore $u_{\varepsilon}$ satisfies

$$
\operatorname{div}\left(a_{i j}(X) \nabla u_{\varepsilon}\right)=\beta_{\varepsilon}\left(u_{\varepsilon}\right), \quad \text { in } \Omega,
$$

in the distributional sense. Each $u_{\varepsilon}$ is a nonnegative function, provided the boundary data $\varphi$ is nonnegative.

Proof. Existence of minimizer as well as the Euler-Lagrange equation associated to the functional follow by classical methods in the Calculus of Variations. Non-negativity of a minimum is obtained as follows. Suppose, for the sake of contradiction, the set $\left\{u_{\varepsilon}<0\right\}$ were not empty. Since $\varphi \geq 0$ on $\partial \Omega$, one sees that $\partial\left\{u_{\varepsilon}<0\right\} \subset\left\{u_{\varepsilon}=0\right\} \cap \Omega$. Since $\beta_{\varepsilon}$ is supported in $[0, \varepsilon]$, from the equation we conclude that $u_{\varepsilon}$ satisfies the homogeneous equation $\operatorname{div}\left(a_{i j}(X) \nabla u_{\varepsilon}\right)=0$ in $\left\{u_{\varepsilon}<0\right\}$. By the maximum principle we conclude $u_{\varepsilon} \equiv 0$ in such a set, which gives a contradiction.

Regarding higher regularity for minimizers, it is possible to show uniform-in- $\varepsilon L^{\infty}$ bounds and also a uniform-in- $\varepsilon C^{0, \alpha}$ estimate, for a universal exponent $0<\alpha<1$.

Theorem 2.2 (Uniform Hölder regularity of minimizers). Fixed a subdomain $\Omega^{\prime} \Subset \Omega$, there exists a constant $C>0$, depending on dimension, ellipticity constants, $\|\varphi\|_{L^{2}}$ and $\Omega^{\prime}$, but independent of $\varepsilon$, such that

$$
\left\|u_{\mathcal{E}}\right\|_{L^{\infty}\left(\Omega^{\prime}\right)}+\left[u_{\varepsilon}\right]_{C^{\alpha}\left(\Omega^{\prime}\right)}<C,
$$


where $0<\alpha<1$ is a universal number.

Proof. The arguments to show Theorem 2.2 follow closely the ones from [2, Theorem 3.4], upon observing that for any ball $B_{r}(Y) \subset \Omega$, there too holds

$$
\int_{B_{r}(Y)} B_{\varepsilon}\left(u_{\varepsilon}\right) d X \leq C r^{n}
$$

for a constant $C$ independent of $\varepsilon$. See also [23, Theorem 4.4] for a result of the same flavor.

As a consequence of Theorem 2.2 up to a subsequence, $u_{\varepsilon}$ converges locally uniformly in $\Omega$ to a nonnegative function $u_{0}$. By linear interpolation techniques, see for instance [22, Theorem 5.4], one verifies that $u_{0}$ is a minimizer of the functional (1.2).

The final result we state in this section gives the sharp lower bound for the grow of $u_{\varepsilon}$ away from $\varepsilon$-level surfaces.

Theorem 2.3 (Linear Growth). Let $\Omega^{\prime} \Subset \Omega$ be a given subdomain and $X_{0} \in \Omega^{\prime} \cap\left\{u_{\varepsilon} \geq\right.$ $\varepsilon\}$ then

$$
u_{\varepsilon}\left(X_{0}\right) \geq c \cdot \operatorname{dist}\left(X_{0}, \partial\left\{u_{\varepsilon} \geq \varepsilon\right\}\right),
$$

where $c$ is a constant that depends on dimension and ellipticity constants, but it is independent of $\varepsilon$.

Proof. The classical proof for linear growth is based on pure energy considerations, combined with a "cutting hole" argument, see for instance [22, Theorem 4.6]. Hence, the same reasoning applied here yields estimate (2.2), with minor modifications.

\section{Lipschitz regularity along the free boundary}

The heart of this work lies in this Section, where we prove that uniform limits of solutions to 2.1) are locally Lipschitz continuous along their free boundaries. We highlight once more that our approach is purely based on the singular partial differential equation satisfied by local minimizers; therefore it can be imported to a number of other contexts, both variational and non-variational.

Theorem 3.1 (Lipschitz regularity). Let $u_{0}$ be a uniform limit point of solutions to

$$
\operatorname{div}\left(a_{i j}(X) \nabla u_{\varepsilon}\right)=\beta_{\varepsilon}\left(u_{\varepsilon}\right) \quad \text { in } \Omega
$$

and assume that $u_{0}(\xi)=0$. Then there exists a universal constant $C>0$, depending only on dimension, ellipticity constants, $\operatorname{dist}(\xi, \partial \Omega)$ and $L^{\infty}$ bounds of the family such that

$$
\left|u_{0}(X)\right| \leq C|X-\xi|
$$

for all point $X \in \Omega$.

Our strategy is based on a flatness improvement argument, within whom the next Lemma plays a decisive role. 
Lemma 3.2. Fixed a ball $B_{r}(Y) \Subset \Omega$ and given $\theta>0$, there exists a $\delta>0$, depending only on $B_{r}(Y)$, dimension, ellipticity constants and $L^{\infty}$ bounds for $u_{\mathcal{E}}$, such that if

$$
\operatorname{div}\left(a_{i j}(X) \nabla u_{\varepsilon}\right)=\delta \cdot \beta_{\varepsilon}\left(u_{\varepsilon}\right)
$$

and

$$
\max \left\{\varepsilon, \inf _{B_{r}(Y)} u_{\varepsilon}\right\} \leq \delta
$$

Then

$$
\sup _{B_{\frac{r}{2}}(Y)} u_{\varepsilon} \leq \theta .
$$

Proof. Let us suppose, for the sake of contradiction, that the Lemma fails to hold. There would then exist a sequence of functions $u_{\varepsilon_{k}}$ satisfying

$$
\operatorname{div}\left(a_{i j}^{k}(X) \nabla u_{\varepsilon_{k}}\right)=\delta_{k} \beta_{\varepsilon_{k}}\left(u_{\varepsilon_{k}}\right)
$$

with $a_{i j}^{k}(\lambda, \Lambda)$-elliptic, $\delta_{k}=\mathrm{o}(1)$, and

$$
\max \left\{\varepsilon_{k}, \inf _{B_{r}(Y)} u_{\varepsilon_{k}}\right\}=: \eta_{k}=\mathrm{o}(1),
$$

but

$$
\sup _{B_{r} / 2(Y)} u_{\varepsilon_{k}} \geq \theta_{0}>0
$$

for some $\theta_{0}>0$ fixed. Let $X_{k}$ be the point where $u_{\varepsilon_{k}}$ attains its minimum in $B_{r}(Y)$ and denote $\sigma:=\operatorname{dist}\left(B_{r}(Y), \partial \Omega\right)>0$. Define the scaled function $v_{k}: B_{\sigma \varepsilon_{k}^{-1}} \rightarrow \mathbb{R}$, by

$$
v_{k}(X):=\frac{u_{\varepsilon_{k}}\left(X_{k}+\varepsilon_{k} X\right)}{\eta_{k}}
$$

One simply verifies that $v_{k} \geq 0$ and it solves, in the distributional sense,

$$
\begin{aligned}
\operatorname{div}\left(a_{i j}^{k}(X) \nabla v_{k}\right) & =\delta_{k} \cdot\left(\frac{\varepsilon_{k}}{\eta_{k}} \beta_{1}\left(\frac{\eta_{k}}{\varepsilon_{k}} v_{k}\right)\right) \\
& =\mathrm{o}(1),
\end{aligned}
$$

as $k \rightarrow \infty$, in the $L^{\infty}$-topology. Also, one easily checks that $v_{k}(0) \leq 1$. Hence, by Harnack inequality, the sequence $v_{k}$ is uniform-in- $k$ locally bounded in $B_{\sigma \varepsilon_{k}^{-1}}(0)$. From De Giorgi, Nash, Moser regularity theory, up to a subsequence, $v_{k}$ converges locally uniformly to an entire $v_{\infty}$. In addition, by standard Caccioppoli energy estimates, the sequence $v_{k}$ is locally bounded in $H^{1}$, uniform in $k$. Also by classical truncation arguments, up to a subsequence, $\nabla v_{k}(X) \rightarrow \nabla v_{\infty}(X)$ a.e. (see [23] and [24] for similar arguments). By ellipticity, passing to another subsequence, if necessary, $a_{i j}$ converges weakly in $L_{\text {loc }}^{2}$ to a $(\lambda, \Lambda)$-elliptic matrix $b_{i j}$. Summarizing we have the following convergences:

$$
\begin{gathered}
v_{k} \rightarrow v_{\infty} \text { locally uniformly in } \mathbb{R}^{n} ; \\
v_{k} \rightarrow v_{\infty} \text { weakly in } H_{\mathrm{loc}}^{1}\left(\mathbb{R}^{n}\right) ; \\
\nabla v_{k}(X) \rightarrow \nabla v_{\infty}(X) \text { almost everywhere in } \mathbb{R}^{n} ; \\
a_{i j}^{k}(X) \rightarrow b_{i j} \text { weakly in } L_{\mathrm{loc}}^{2}\left(\mathbb{R}^{n}\right) .
\end{gathered}
$$


Our next step is to the pass the limits above aiming to conclude that

$$
\operatorname{div}\left(b_{i j}(X) \nabla v_{\infty}\right)=0, \quad \text { in } \mathbb{R}^{n} .
$$

This is a fairly routine procedure, but we will carry it out for the sake of the readers. Given a test function $\phi \in C_{0}^{1}\left(\mathbb{R}^{n}\right)$, let $k_{0} \in \mathbb{N}$ be such that $B_{\sigma \varepsilon_{k}^{-1}} \supset \operatorname{Supp} \phi:=K$. For $k>k_{0}$, we define the integrals

$$
\begin{aligned}
\mathcal{J}_{k}^{1} & :=\int_{K}\left\langle a_{i j}^{k}(X) \nabla v_{k}, \nabla \phi\right\rangle d X ; \\
\mathcal{J}_{k}^{2} & :=\int_{K}\left\langle a_{i j}^{k}(X) \cdot\left(\nabla v_{\infty}-\nabla v_{k}\right), \nabla \phi\right\rangle d X ; \\
\mathcal{J}_{k}^{3} & :=\int_{K}\left\langle\left(b_{i j}-a_{i j}^{k}\right)(X) \cdot \nabla v_{\infty}, \nabla \phi\right\rangle d X ;
\end{aligned}
$$

and write

$$
\int_{\mathbb{R}^{n}}\left\langle b_{i j}(X) \nabla v_{\infty}, \nabla \phi\right\rangle d X=\mathcal{J}_{k}^{1}+\mathcal{J}_{k}^{2}+\mathcal{J}_{k}^{3} .
$$

The purpose is to show that

$$
\lim _{k \rightarrow \infty} \mathcal{J}_{k}^{1}+\mathcal{J}_{k}^{2}+\mathcal{J}_{k}^{3}=0
$$

For that, let $\gamma>0$ be a given number small, positive number. It follows straight from (3.2) that $\mathcal{J}_{k}^{1}=\mathrm{o}(1)$ as $k \rightarrow \infty$. From (3.6), we also have straightly that $\mathcal{J}_{k}^{3}=\mathrm{o}(1)$ as $k \rightarrow \infty$. Hence, for $k_{1} \geq k_{0}$, we have

$$
\left|\mathcal{J}_{k}^{1}\right|+\left|\mathcal{J}_{k}^{3}\right| \leq \frac{\gamma}{2}
$$

Let us now analyze the convergence of $\mathcal{J}_{k}^{2}$. It follows from (3.5) and Ergorov's theorem, that there exists a compact set $\tilde{K} \subset K$, such that

$$
\int_{K \backslash \tilde{K}}|\nabla \phi| d X \leq \frac{\gamma}{5 \Lambda \sup _{k}\left\|\nabla v_{k}\right\|_{2}}
$$

and $k_{2} \geq k_{1}$ such that

$$
\left|\nabla v_{k}(X)-\nabla v_{\infty}(X)\right| \leq \frac{3 \gamma}{5 \Lambda\|\nabla \phi\|_{2} \mathcal{L}^{n}(K)},
$$

in $\tilde{K}$, for all $k \geq k_{2}$. Hence, for $k \geq k_{2}$, we estimate, breaking it into two integrals on $\tilde{K}$ and on $K \backslash \tilde{K}$, and using Hölder inequality, finally obtain

$$
\left|\mathcal{J}_{k}^{2}\right| \leq \frac{\gamma}{2}
$$

We have henceforth proven the aimed convergence which gives (3.7). Applying Liouville theorem to $v_{\infty}$, we conclude that

$$
v_{\infty} \equiv \text { Const. }<+\infty,
$$

for a bounded constant, in the whole space. The corresponding limiting function $u_{\infty}$ obtained from $u_{\varepsilon_{k}}$ must therefore be identically zero. We now reach a contradiction with (3.1) for $k \gg 1$. The Lemma is proven. 
Before continuing, we remark that if $u_{\varepsilon}$ is a solution to the original equation (2.1) and a positive number $\bar{\delta}>0$ is given, then the zoomed-in function

$$
\tilde{u}_{\varepsilon}(X)=u_{\varepsilon}(\sqrt{\bar{\delta}} X)
$$

satisfies in the distributional sense the equation

$$
\operatorname{div}\left(\tilde{a}_{i j}(X) \nabla \tilde{u}_{\varepsilon}\right)=\bar{\delta} \beta_{\varepsilon}\left(\tilde{u}_{\varepsilon}\right),
$$

where $\tilde{a}_{i j}(X)=a_{i j}(\sqrt{\bar{\delta}} X)$ is another $(\lambda, \Lambda)$-elliptic matrix.

We are in position to start delivering the proof of Theorem 3.1. Let $u_{\varepsilon}$ be a bounded sequence of distributional solutions to 2.1) and $u_{0}$ a limit point in the uniform convergence topology. We assume, with no loss, that $\xi=0$, that is $u_{0}(0)=0$. Within the statement of Lemma3.2, select

$$
\theta=\frac{1}{2}
$$

Since $u_{\varepsilon}(0) \rightarrow 0$ as $\varepsilon \rightarrow 0$, Lemma 3.2 together with the above remark, gives the existence of a positive, universal number $\delta_{\star}>0$, such that if $0<\varepsilon \leq \varepsilon_{0} \ll 1$, for $\tilde{u}_{\varepsilon}(X):=u_{\varepsilon}\left(\sqrt{\delta_{\star}} X\right)$ we have

$$
\sup _{B_{1 / 2}} \tilde{u}_{\varepsilon}(X) \leq \frac{1}{2} .
$$

Passing to the limit as $\varepsilon \rightarrow 0$, we obtain

$$
\sup _{\substack{\frac{\sqrt{\delta_{\star}}}{2}}} u_{0}(X) \leq \frac{1}{2} .
$$

Define, in the sequel, the rescaled function

$$
v^{1}(X):=2 u_{\varepsilon}\left(\frac{\sqrt{\delta_{\star}}}{2} X\right)
$$

It is simple to verify that $v^{1}$ satisfies

$$
\operatorname{div}\left(a_{i j}^{1}(X) \nabla v^{1}(X)\right)=\delta_{\star} \beta_{2 \varepsilon}\left(v^{1}\right),
$$

in the distributional sense, where $a_{i j}^{1}(X)=a_{i j}\left(\sqrt{\delta_{\star}} X / 2\right)$ is another $(\lambda, \Lambda)$-elliptic matrix. Once more, $v^{1}(0) \rightarrow 0$ as $\varepsilon \rightarrow 0$, hence, for $\varepsilon \leq \varepsilon_{1}<\varepsilon_{0} \ll 1$, we can apply Lemma 3.2 to $v^{1}$ and deduce, after scaling the inequality back,

$$
\sup _{\frac{\sqrt{\delta_{\star}}}{4}} u_{0}(X) \leq \frac{1}{4} .
$$

Continuing this process inductively, we conclude that for any $k \geq 1$, that holds

$$
\sup _{\frac{\sqrt{\delta_{\star}}}{2^{k}}} u_{0}(X) \leq \frac{1}{2^{k}} .
$$


Finally, given $X \in B_{1 / 2}$ let $k \in \mathbb{N}$ be such that

$$
\frac{\sqrt{\delta_{\star}}}{2^{k+1}}<|X| \leq \frac{\sqrt{\delta_{\star}}}{2^{k}}
$$

We estimate from 3.9

$$
\begin{aligned}
u_{0}(X) & \leq \sup _{B} u_{0}(X) \\
& \leq \frac{1}{2^{\delta_{\star}}} \\
& \leq \frac{2}{\sqrt{\delta_{\star}}}|X|,
\end{aligned}
$$

and the proof of Theorem 3.1 is concluded.

Obviously, the (improved) regularity estimate granted by Theorem 3.1 holds solely along the free boundary. For any point $Z \in\{u>0\}$, the best estimate available drops back to $C^{0, \alpha}$, for some unknown $0<\alpha$, strictly less than one. The question we would like to answer now is what is the minimum organization required on the medium so that solutions to the cavitation problem is locally Lipschitz continuous, up to the free boundary.

Definition 3.3. Given a large constant $K>0$, we say that a uniform elliptic matrix $a_{i j}(X)$ satisfies ( $K$-Lip) property if for any $0<d<1$ and any $h \in H^{1}\left(B_{d}\right)$ solving

$$
\operatorname{div}\left(a_{i j}(X) \nabla h\right)=0 \text { in } B_{d}
$$

in the distributional sense, there holds

$$
\|\nabla h\|_{L^{\infty}\left(B_{d / 2}\right)} \leq \frac{K}{d} \cdot\|h\|_{L^{\infty}\left(B_{d}\right)} .
$$

It is classical that Dini continuity of the medium is enough to assure that $a_{i j}$ satisfies ( $K$-Lip) property, for some $K>0$ that depends only upon dimension, ellipticity constants and the Dini-modulus of continuity of $a_{i j}$. Indeed under Dini continuity assumption on $a_{i j}$, distributional solutions are of class $C^{1}$.

Our next Corollary says that uniform limits of singularly perturbed equation (2.1) is Lipschitz continuous, up to the free boundary provided $a_{i j}$ satisfies ( $K$-Lip) property for some $K>0$. The (by no means obvious) message being that when it comes to Lipschitz estimates, the homogeneous equation and the free boundary problem $\operatorname{div}\left(a_{i j}(X) \nabla u\right) \sim$ $\delta_{0}(u)$ require the same amount of organization of the medium.

Corollary 3.4. Under the assumptions of Theorem 3.1 assume further that $a_{i j}(X)$ satisfies (K-Lip) property for some $K$. Then, given a subdomain $\Omega^{\prime} \Subset \Omega$,

$$
\left|\nabla u_{0}(X)\right| \leq C
$$

for a constant that depends only on dimension, ellipticity constants, $\operatorname{dist}\left(\partial \Omega^{\prime}, \partial \Omega\right), L^{\infty}$ bounds of the family and $K$. 
Proof. It follows from Theorem 3.1 and property $K$ that $u_{0}$ is pointwise Lipschitz continuous, i.e.,

$$
\left|\nabla u_{0}(\xi)\right| \leq C(\xi)
$$

We have to show that $C(\xi)$ remains bounded as $\xi$ goes to the free boundary. For that, let $\xi$ be a point near the free boundary $\partial\left\{u_{0}>0\right\}$ and denote by $Y \in \partial\left\{u_{0}>0\right\}$ a point such that

$$
|Y-\xi|=: d=\operatorname{dist}\left(\xi, \partial\left\{u_{0}>0\right\}\right) .
$$

From Theorem 3.1, we can estimate

$$
\sup _{B_{d / 2}(\xi)} u_{0}(\xi) \leq \sup _{B_{2 d}(Y)} u_{0}(\xi) \leq C \cdot 2 d
$$

Applying ( $K$-Lip) property to the ball $B_{d / 2}(\xi)$, we obtain

$$
\left|\nabla u_{0}(\xi)\right| \leq \frac{2 K}{d} \cdot 2 C d=4 C \cdot K
$$

and the proof is concluded.

\section{Lipschitz estimates for the minimization problem}

Limiting functions $u_{0}$ obtained as $\varepsilon$ goes to zero from a sequence $u_{\varepsilon}$ of minimizers of functional (1.6) are minima of the discontinuous functional (1.2). Hence, limiting minima are Lipschitz continuous along their free boundaries. Nonetheless, as previously advertised in Theorem 1.1, the sharp Lipschitz regularity estimate holds indeed for any minima of the functional (1.2), not necessarily for limiting functions.

In this intermediate Section we shall comment on how one can deliver this estimate directly from the analysis employed in the proof of Theorem 3.1. In fact, the proof of Lipschitz estimate for minima of the functional (1.2) is simpler than the proof delivered in previous section, which has been based solely on the singular equation satisfied. When a minimality property is available, the arguments can be rather simplified. For instance, strong minimum principle holds for local minima but is no longer available for a generic critical point. This is part of the reason why the arguments from previous section had be be based on blow-ups and Liouville theorem.

Theorem 4.1. Let $u_{0} \geq 0$ be a minimum to

$$
\mathcal{F}(u)=\int_{\Omega}\left\{\frac{1}{2}\left\langle a_{i j}(X) \nabla u, \nabla u\right\rangle+\chi_{\{u>0\}}\right\} d X
$$

and assume that $u_{0}(\xi)=0$. Then there exists a universal constant $C>0$, depending only on dimension, ellipticity constants, $\operatorname{dist}(\xi, \partial \Omega)$ and its $L^{\infty}$ norm such that

$$
u_{0}(X) \leq C|X-\xi|,
$$

for all point $X \in \Omega$. 
The proof follows the lines designed in Section 3. We obtain the corresponding flatness Lemma as follows:

Lemma 4.2. Fixed a ball $B_{r}(Y) \Subset \Omega$ and given $\theta>0$, there exists a $\delta>0$, depending only on $B_{r}(Y)$, dimension, ellipticity constants and $L^{\infty}$ norm of $u_{0}$, such that if $u_{0}$ is a nonnegative minimum of

$$
\mathcal{F}^{\delta}(u)=\int_{\Omega}\left\{\frac{1}{2}\left\langle a_{i j}(X) \nabla u, \nabla u\right\rangle+\delta \cdot \chi_{\{u>0\}}\right\} d X,
$$

and $u_{0}(Y)=0$, then

$$
\sup _{B_{\frac{r}{2}}(Y)} u_{0} \leq \theta .
$$

Proof. The proof follows by a similar tangential analysis of the proof of Lemma 3.2 , but in fact in a simpler fashion. The tangential functional, obtained as $\delta \rightarrow 0$, satisfies minimum principle, hence the limiting function, from the contradiction argument, must be identically zero.

Here are some details: suppose, for the sake of contradiction, that the Lemma fails to hold. It means, for a sequence $(\lambda, \Lambda)$-elliptic matrices, $a_{i j}^{k}$, and a sequence of minimizers $u_{k}$ of

$$
\mathcal{F}^{k}(u)=\int_{\Omega}\left\{\frac{1}{2}\left\langle a_{i j}^{k}(X) \nabla u, \nabla u\right\rangle+\delta_{k} \cdot \chi_{\{u>0\}}\right\} d X,
$$

with $\delta_{k}=\mathrm{o}(1)$, and, say $\left\|u_{k}\right\|_{\infty} \leq 1$,

$$
\sup _{B_{r} / 2(Y)} u_{k} \geq \theta_{0}>0
$$

for some $\theta_{0}>0$ fixed. As in Lemma 3.2 by compactness, up to a subsequence, $u_{k} \rightarrow$ $u_{0}$. Passing the limits we conclude $u_{0}$ is a local minimum of

$$
\mathcal{F}^{\infty}(u)=\int \frac{1}{2}\left\langle b_{i j}(X) \nabla u_{0}, \nabla u_{0}\right\rangle d X .
$$

Since, $u_{0} \geq 0$ and $u_{0}(Y)=0$, by the strong minimum principle, see for instance [14, Theorem 7.12], $u_{0} \equiv 0$. We now reach a contradiction with (4.1) for $k \gg 1$. The Lemma is proven.

Once we have obtained Lemma 4.2, the proof of Theorem 4.1 follows exactly as the final steps in the proof of Theorem 3.1

\section{Gradient control in two-phase problems}

In this Section we show that Theorem 3.1 as well as Theorem 4.1 hold for two-phase problems, provided a one-side control is a priori known. It is interesting to compare this with the program developed in [4, 5, 6], where monotonicity formula yields similar conclusion. 
Let us briefly comment on such generalization, in the (simpler) minimization problem. The singular perturbed one can be treated similarly. We start by placing the negative values of $u$ within a universally controlled slab, i.e.:

$$
\inf _{\Omega} u \geq-\delta_{\star}
$$

for a universal value $\delta_{\star}>0$. Such a condition is realistic for models involving very low temperatures, i.e., for physical problem near the absolute zero for thermodynamic temperature (zero Kelvin). A scaling of the problem places any solution into this setting. Within the proof of Lemma 4.2, one includes condition (5.1) in the compactness argument. Here is the two-phase version of Lemma 4.2

Lemma 5.1. Fixed a ball $B_{r}(Y) \Subset \Omega$ and given $\theta>0$, there exists $a \delta>0$, depending only on $B_{r}(Y)$, dimension, ellipticity constants and $L^{\infty}$ norm of $u$, such that if $u$ is a changing sign minimum of

$$
\mathcal{F}^{\tilde{\delta}}(u)=\int_{\Omega}\left\{\frac{1}{2}\left\langle a_{i j}(X) \nabla u, \nabla u\right\rangle+\tilde{\delta} \cdot \chi_{\{u>0\}}\right\} d X,
$$

for $\tilde{\delta} \leq \delta$, with

$$
u_{0}(Y)=0 \quad \text { and } \quad \inf _{\Omega} u \geq-\delta
$$

then

$$
\sup _{B_{\frac{r}{2}}(Y)}|u| \leq \theta .
$$

The proof of Lemma5.1 follows the lines of Lemma 4.2 noticing that, by letting $\delta=\mathrm{o}(1)$ in the compactness approach, the tangential configuration is too a nonnegative minima of a functional which satisfies minimum principle.

Theorem 5.2. Let $u_{0}$ be a sign changing minimum of the functional

$$
\mathcal{F}(u)=\int_{\Omega}\left\{\frac{1}{2}\left\langle a_{i j}(X) \nabla u, \nabla u\right\rangle+\chi_{\{u>0\}}\right\} d X,
$$

with $u_{0}(\xi)=0,-1 \leq u_{0} \leq 1$. Assume $u^{-}$is Lipschitz continuous at 0 . Then $u^{+}$(and therefore $u$ ) is too Lipschitz at 0 and

$$
|\nabla u(0)| \leq C\left|\nabla u^{-}(0)\right| .
$$

Proof. We can assume, with no loss, $\Omega=B_{2}$ and $\xi=0$. By universal continuity estimate, Theorem 2.2, we can choose a universal number $0<\tau_{0} \ll 1$, such that the function $v: B_{1} \rightarrow \mathbb{R}$, given by

$$
v(X):=u\left(\tau_{0} X\right),
$$

satisfies the hypothesis of Lemma5.1, for $\theta=\frac{1}{2}$. Selecting $0<\tau_{0} \ll\left|\nabla u^{-}(0)\right|^{-1}$, even smaller if necessary, we can assure

$$
\left|\nabla v^{-}(0)\right| \leq \delta_{1 / 2},
$$


where $\delta_{1 / 2}$ is the number from Lemma 5.1 when we take $\theta=\frac{1}{2}$. Define in the sequel $v_{2}: B_{1} \rightarrow \mathbb{R}$ by

$$
v_{2}(X):=2 v\left(\frac{1}{2} X\right) .
$$

Clearly $v_{2}$ is a minimum of a functional $\mathcal{F}^{\tilde{\delta}}$ for $\tilde{\delta} \leq \delta_{1 / 2}, v_{2}(0)=0$, and by Lemma 5.1 it also verifies $-1 \leq v_{2} \leq 1$. We estimate

$$
\inf _{B_{1}} v_{2} \geq-\left|\nabla v^{-}(0)\right| \geq-\delta_{1 / 2} .
$$

Hence, $v_{2}$ is also within the hypothesis of Lemma 5.1 Carrying the induction process shows that

$$
\sup _{B_{\tau_{0} 2^{-k}}}|u| \leq 2^{-k}
$$

Now, given $0<r \ll 1$, we choose $k \in \mathbb{N}$ such that $\tau_{0} 2^{-(k+1)} \leq r \leq \tau_{0} 2^{-k}$ and compute

$$
\sup _{B_{r}}|u| \leq \sup _{B_{\tau_{0} 2^{-k}}}|u| \leq 2^{-k} \leq \frac{2}{\tau_{0}} r .
$$

The Theorem is proven.

Similarly, one can use these set of ideas when a density control of the negative phase is given. For instance if

$$
\mathcal{L}^{n}\left(\{u<0\} \cap B_{r}\right) \leq \delta_{\star} r^{n},
$$

where $\delta_{\star} \ll 1$ is universally small, then Lipschitz regularity along the free boundary holds. Indeed, as before, one could add the constrain (5.2) within the compactness approach, letting $\delta_{\star}=\mathrm{o}(1)$, and the limiting configuration is too a nonnegative function. Now, within the induction procedure, condition (5.2) scales properly, in the sense that at each scale, condition (5.2) holds with the same initial constant $\delta_{\star}$. Compare for instance with [17].

\section{Geometric estimates of the free boundary}

In this Section we show how the improved estimate given by Theorem 3.1 (or else Theorem 4.10 implies some geometric estimates on the free boundary. Hereafter in this Section, $u_{0} \geq 0$ will always denote a limit point obtained from a sequence of minimizers of the functional (1.6). We will denote by $\Omega_{0}$ the non coincidence set, $\Omega_{0}:=\left\{u_{0}>\right.$ $0\} \cap \Omega$. Unless otherwise stated, no continuity assumption is imposed upon the medium $a_{i j}$.

Theorem 6.1 (Nondegeneracy). Let $\Omega^{\prime} \Subset \Omega$ be a given subdomain and $Y \in \Omega^{\prime} \cap$ $\overline{\left\{u_{0}>0\right\}}$ then

$$
\sup _{B_{r}(Y)} u_{0} \geq c \cdot r
$$

for $r<\operatorname{dist}\left(\Omega^{\prime}, \partial \Omega\right)$. 
Proof. Letting $\varepsilon \rightarrow 0$ in Theorem 2.3 we conclude $u_{0}$ grow linearly away from the free boundary. Owning Lipchitz regularity along $\partial\left\{u_{0}>0\right\} \cap \Omega^{\prime}$, Theorem 3.1 we can then perform a polygonal type of argument a la Caffarelli, see for instance [21, Lemma 4.2.7], to establish such a strong non-degeneracy estimate.

Theorem 6.2. Given a subdomain $\Omega^{\prime} \Subset \Omega$, there exists a constant $\theta>0$, such that if $X_{0} \in \partial \Omega_{0}$ is a free boundary point then

$$
\mathscr{L}^{n}\left(\Omega_{0} \cap B_{r}\left(X_{0}\right)\right) \geq \theta r^{n},
$$

for all $0<r<\operatorname{dist}\left(\partial \Omega^{\prime}, \partial \Omega\right)$. Furthermore there is a universal constant $0<\varsigma \leq 1$ such that

$$
\operatorname{dim}_{\mathscr{H}}\left(\partial \Omega_{0}\right) \leq n-\varsigma,
$$

where $\operatorname{dim}_{\mathscr{H}}(E)$ means the Hausdorff dimension to the set $E$.

Proof. It follows readily from non-degeneracy property, Theorem 6.1, there exists a point $\xi_{r} \in \partial B_{r}\left(X_{0}\right)$ such that

$$
u_{0}\left(\xi_{r}\right) \geq c r,
$$

for a constant $c>0$ depending only on the data of the problem. Now, for $0<\mu \ll 1$, small enough, there holds

$$
B_{\mu r}\left(\xi_{r}\right) \subset \Omega_{0} .
$$

Indeed, one simply verifies that if

$$
B_{\mu r}\left(\xi_{r}\right) \cap \partial\left\{u_{0}>0\right\} \neq \emptyset,
$$

then from Theorem 3.1 we can estimate

$$
c r \leq u_{0}\left(\xi_{r}\right) \leq \sup _{B_{\mu r}\left(Z_{0}\right)} u_{0} \leq C \mu r
$$

which is a lower bound for $\mu$. Hence, if $\mu<c \cdot C^{-1}$, 6.1 must hold. Now, with such $\mu>0$ fixed, we estimate

$$
\mathcal{L}^{n}\left(B_{r}\left(X_{0}\right) \cap \Omega_{0}\right) \geq \mathcal{L}^{n}\left(B_{r}\left(X_{0}\right) \cap B_{\mu r}\left(\xi_{r}\right)\right) \geq \theta r^{n}
$$

and the uniform positive density is proven.

Let us turn our attention to the Hausdorff dimension estimate. Given $\sigma=X_{0}$ in $\partial\{u>0\}$, we choose

$$
\sigma \prime=t \xi_{r}+(1-t) X_{0}
$$

with $t$ close enough to 1 as to

$$
B_{\frac{1}{2} \mu \cdot r}(\sigma \prime) \subset B_{\mu}\left(\xi_{r}\right) \cap B_{r}(\sigma) \subset B_{r}(\sigma) \backslash \partial\{u>0\} .
$$

We have verified $\partial\{u>0\} \cap B_{1 / 2}$ is $(\mu / 2)$-porous, hence by a classical result, see for instance [15, Theorem 2.1], its Hausdorff dimension is at most $n-C \mu^{n}$, for a dimensional constant $C>0$. 
For problems modeled in a merely measurable medium, one should not expect an improved Hausdorff estimate for the free boundary. When diffusion is governed by the Laplace operator, then Alt-Caffarelli theory gives that $\varsigma=1$. A natural question is what is the minimum organization of the medium as to obtain perimeter estimates of the free boundary. Next Theorem gives an answer to that issue.

Theorem 6.3. Assume $a_{i j}$ satisfy (K-Lip) property for some $K>0$. Then the free boundary has local finite perimeter. In particular $\operatorname{dim}_{\mathscr{H}}\left(\partial \Omega_{0}\right)=n-1$.

Proof. Fixed a free boundary point $X_{0} \in \partial \Omega_{0}$ and given a small, positive number $\mu$ one checks that

$$
\int_{\left\{0<u_{0}<\mu\right\} \cap B_{r}\left(X_{0}\right)}\left|\nabla u_{0}\right|^{2} \leq C \mu r^{n-1} .
$$

This is obtained by integration by parts and Lipschitz estimate on $B_{r}$. In the sequel, we compare the left hand side of (6.2) with $\left|\left\{0<u_{0}<\mu\right\} \cap B_{r}\left(X_{0}\right)\right|$. This is done by considering a finite overlapping converging, $\left\{B_{j}\right\}$, of $\partial \Omega_{0}$ by balls of radius proportional to $\mu$ and centered on $\partial \Omega_{0} \cap B_{r}\left(X_{0}\right)$. In each ball $B_{j}$, we can find subballs $B_{j}^{1}, B_{j}^{2}$ with the radii $\sim \mu$, such that

$$
u_{0} \geq \frac{3}{4} \mu \text { in } B_{j}^{1} \quad \text { and } \quad u_{0} \leq \frac{2}{3} \mu \text { in } B_{j}^{2} .
$$

Existence of such balls is obtained by nondegeneracy property followed by Poincaré inequality. Now, for $\mu \ll r$, we have

$$
B_{r}\left(X_{0}\right) \cap\left\{0<u_{0}<\mu\right\} \subset \bigcup 2 B_{j} \subset B_{4 r}\left(X_{0}\right) .
$$

Finally, if we call $A:=\left\{0<u_{0}<\mu\right\}$, the above gives

$$
\begin{aligned}
& \int_{B_{4 r}\left(X_{0}\right) \cap A}\left|\nabla u_{0}\right|^{2} d X \geq \int_{\left(\cup 2 B_{j}\right) \cap A}\left|\nabla u_{0}\right|^{2} d X \\
& \geq \frac{1}{m} \sum \int_{2 B_{j} \cap A}\left|\nabla u_{0}\right|^{2} d X \\
& \geq c \sum \mathcal{L}^{n}\left(B_{j}\right) \\
& \geq c \mathcal{L}^{n}\left(B_{r}\left(X_{0}\right) \cap A\right),
\end{aligned}
$$

where $m$ is the total number of balls, which can be taken universal, by Heine-Borel's Theorem. Combining the above estimate with (6.2), gives

$$
\mathcal{L}^{n}\left(\left\{0<u_{0}<\mu\right\} \cap B_{r}\left(X_{0}\right)\right) \leq C \mu r^{n-1},
$$

which implies the desired Hausdorff estimate by classical considerations. For further details, see for instance [21, Chapter 4]. 


\section{References}

[1] Alt, H. W.; Caffarelli, L. A. Existence and regularity for a minimum problem with free boundary. J. Reine Angew. Math. 325 (1981), 105-144.

[2] Amaral, Marcelo D.; Teixeira, Eduardo V., Free transmission problem. Comm. Math. Phys. 337 (2015), no. 3, 1465-1489.

[3] H. Berestycki, L. A. Caffarelli and L. Nirenberg, Uniform estimates for regularization of free boundary problems. Analysis and partial differential equations, 567-619, Lecture Notes in Pure and Appl. Math., 122, Dekker, New York, 1990.

[4] Caffarelli, L. A. A Harnack inequality approach to the regularity of free boundaries. I. Lipschitz free boundaries are $C^{1, \alpha}$.Rev. Mat. Iberoamericana 3 (1987), no. $2,139-162$.

[5] Caffarelli, L. A. A Harnack inequality approach to the regularity of free boundaries. II. Flat free boundaries are Lipschitz. Comm. Pure Appl. Math. 42 (1989), no. $1,55-78$.

[6] Caffarelli, L. A. A Harnack inequality approach to the regularity of free boundaries. III. Existence theory, compactness, and dependence on X. Ann. Scuola Norm. Sup. Pisa Cl. Sci. (4) 15 (1988), no. 4, 583-602 (1989).

[7] Caffarelli, L.A., Lee, K.-A., Mellet, A.S. Limit and homogenization for flame propagation in periodic excitable media. Arch. Ration. Mech. Anal. 172 (2), 153190 (2004)

[8] Caffarelli, L. A.; Lederman, C.; Wolanski, N. Uniform estimates and limits for a two phase parabolic singular perturbation problem. Indiana Univ. Math. J. 46 (1997), no. 2, 453-489.

[9] Caffarelli, L. A.; Lederman, C.; Wolanski, N. Pointwise and viscosity solutions for the limit of a two phase parabolic singular perturbation problem. Indiana Univ. Math. J. 46 (1997), no. 3, 719-740.

[10] L. A. Caffarelli and S. Salsa, A geometric approach to free boundary problems, Graduate Studies in Mathematics, vol. 68, American Mathematical society, Providence, RI, 2005

[11] Caffarelli,L.A.,Vazques, J.L. A free boundary problem for the heat equation arising in flame propagation. Trans. Am. Math. Soc. 347, 411-441 (1995)

[12] Danielli, D; Petrosyan, A. A minimum problem with free boundary for a degenerate quasilinear operator. Calc. Var. Partial Differential Equations 23 (2005), no. $1,97-124$.

[13] Danielli, D.; Petrosyan, A., Shahgholian, H. A singular perturbation problem for the p-Laplace operator. Indiana Univ. Math. J. 52, (2003) 457-476. 
[14] Giusti, Enrico Direct methods in the calculus of variations. World Scientific Publishing Co., Inc., River Edge, NJ, 2003. viii+403 pp. ISBN: 981-238-043-4

[15] Koskela, Pekka; Rohde, Steffen Hausdorff dimension and mean porosity. Math. Ann. 309 (1997), no. 4, 593-609.

[16] Leitão, Raimundo; Teixeira, Eduardo V. Regularity and geometric estimates for minima of discontinuous functionals. Rev. Mat. Iberoam. 311 (2015) 69-108.

[17] Lee, Ki-Ahm; Shahgholian, Henrik Regularity of a free boundary for viscosity solutions of nonlinear elliptic equations. Comm. Pure Appl. Math. 54 (2001), no. $1,43-56$.

[18] Moreira, D.; Teixeira, Eduardo V. A singular perturbation free boundary problem for elliptic equations in divergence form. Calc. Var. Partial Differential Equations, 29 (2007), no 2, 161-190.

[19] Ricarte, G.; Teixeira, Eduardo V. Fully nonlinear singularly perturbed equations and asymptotic free boundaries. J. Funct. Anal., 261, no 6 (2011), 1624-1673.

[20] Rossi, J.; Teixeira, Eduardo V.; Urbano, J.M. Optimal regularity at the free boundary for the infinity obstacle problem. Interfaces Free Bound. 7 (2015), 381-398.

[21] Teixeira, Eduardo V., Elliptic regularity and free boundary problems: an introduction, Publicações Matemáticas do IMPA. 26o Colóquio Brasileiro de Matemática. Instituto Nacional de Matemática Pura e Aplicada (IMPA), Rio de Janeiro, 2007. ii+205 pp. ISBN: 978-85-244-0252-4

[22] Teixeira, Eduardo V. A variational treatment for elliptic equations of the flame propagation type: regularity of the free boundary. Ann. Inst. H. Poincaré Anal. Non Linéaire, 25 (2008), pp. 633-658.

[23] Teixeira, Eduardo V. Optimal design problems in rough inhomogeneous media. Existence theory. Amer. J. Math., Vol 132, No 6 (2010) pp. 1445-1492.

[24] Teixeira, Eduardo V. Sharp regularity for general Poisson equations with borderline sources. J. Math. Pures Appl. (9) 99 (2013), no. 2, 150-164.

[25] Teixeira, Eduardo V. Regularity for quasilinear equations on degenerate singular sets. Math. Ann. 358 (2014), no 1, 241-256.

[26] Teixeira, Eduardo V. Regularity for the fully nonlinear dead-core problem. To appear in Math. Ann. DOI 10.1007/s00208-015-1247-3

EDUARDO V. TEIXEIRA

Universidade Federal do Ceará

Departamento de Matemática

Campus do Pici - Bloco 914

Fortaleza, CE - Brazil

teixeira@mat.ufc.br
DISSON DOS PRAZERES

Center for Mathematical Modeling Universidad de Chile Beauchef 851, Edificio Norte - Piso 7

Santiago, Chile

dsoaresdim.uchile.cl 